\title{
The Alteration of Subtelomeric DNA Methylation in Aging-Related Diseases
}

\author{
Haochang Hu, Bin Li and Shiwei Duan* \\ Medical Genetics Center, School of Medicine, Ningbo University, Ningbo, China
}

The telomere is located at the end of the chromosome and consists of a non-coding, repetitive DNA sequence. As the cell divides, the length of telomere gradually decreases. A very short telomere can terminate mitosis, and thus telomere length becomes a hallmark of cellular aging. The $500 \mathrm{~kb}$ region of each autosomal arm terminal is the so-called subtelomeric region. Both telomere and subtelomere have high-density DNA repeats. Telomeres do not contain genes or CpG sequences, while subtelomeres contain small amounts of genes and high-density CpG sequences, and DNA methylation often occurs in subtelomeres. Previous studies have shown that aberrant methylation of subtelomeric DNA exists in many diseases, and it has a certain effect on the regulation of telomere length. In this review, we focus on the correlation between subtelomeric DNA methylation and aging-related diseases. We also summarize the relationship between subtelomeric methylation and telomere length in different diseases.

Keywords: epigenetic modification, subtelomeric DNA methylation, telomere length, telomerase, age-related disease

\section{INTRODUCTION}

In the 1930s, people began to understand telomere, a special structure on the chromosome. With continued cell division, telomeres gradually shorten, and a very short telomere terminates mitosis (Harley et al., 1990). When a cell stops replicating, it enters a period called "cell decay," and thus telomere length becomes a hallmark of cellular aging. In addition, the detection of telomere length can also help us assess the risk of aging disease (Herrmann et al., 2018).

DNA methylation is prevalent in the subtelomeric DNA region of mammals. This epigenetic phenomenon may play an important role in transcriptional regulation and chromosomal structural remodeling by regulating DNA binding factors.

Recent studies have shown that subtelomeric DNA methylation changes in many diseases, which are closely related to telomere length control mechanisms. In this review we focus on the correlation of subtelomeric DNA methylation with aging-related diseases. We also check the relationship between subtelomeric methylation and telomere length in different diseases.

Accepted: 12 December 2018

Published: 09 January 2019

Citation:

Hu H, Li B and Duan S (2019) The Alteration of Subtelomeric DNA

Methylation in Aging-Related Diseases. Front. Genet. 9:697.

doi: 10.3389/fgene.2018.00697

\section{TELOMERES, TELOMERASE ACTIVITY AND TELOMERE LENGTH}

Telomere was first discovered by Hermann in 1938, and it is a nuclear protein structure at the end of the chromosome (Herrmann et al., 2018). Telomeres are protective caps at the ends of 
chromosomes in cells, and they are composed of non-coding, repetitive DNA sequences and telomere-binding proteins. Telomere DNA sequences are both highly conserved and speciesspecific, and each organism has its own specific sequence and average length. The human and mammalian telomere sequence is -TTAGGG- (Steinert et al., 2004). The telomere-binding protein is a complex of six proteins named shelterin that consists of TRF1, TRF2, TIN2, Rap1, TPP1, and POT1. The complex not only prevents the activation of the DNA damage reaction at the end of the chromosome, but also regulates the telomerase activity at the end of the chromosome, thereby maintaining the stability of the T-loop structure. The T-loop structure is the single-stranded fold protruding at the $3^{\prime}$ end and invading the telomere double helix structure. This structure facilitates the formation of a cap structure at the end of the chromosome by the telomere (Figure 1). Telomere dysfunction leads to the fusion of the ends of the chromosome, leading to chromosomal instability (Oh et al., 2011).

Telomerase is a special ribonucleoprotein protease complex with reverse transcriptase activity. It uses a primer-specific recognition site to synthesize telomeric DNA at the end of the chromosome and extends its telomere with its own RNA as a template. Telomere repeat amplification protocol (TRAP) assay is a commonly used routine assay with high sensitivity for telomerase activity (Kim et al., 1994). Due to PCR inhibition by biomolecules such as bile salt, hemoglobin, heparin, and lactoferrin, TRAP assay is prone to false-negative results (Kim and $\mathrm{Wu}, 1997)$. Although other telomerase assays based on various sensors or methods are developed, these assays are less sensitive than TRAP assay (Yaku et al., 2013). Fortunately, the integration of TRAP internal controls helps to measure telomerase activity levels, and the false negative results of telomerase-positive samples are extremely low (Kim and $\mathrm{Wu}$, 1997). Telomerase assay with high sensitivity and low false negative results should be explored in the future.

The length of telomeres can be thought as the individual's biological clock. There are different strategies for determining telomere length, including terminal restriction fragment (TRF), Southern blotting (SB) analysis, quantitative polymerase chain reaction (PCR), and flow-fluorescence in situ hybridization (FISH) (Tarik et al., 2018). TRF analysis is the oldest approach for determining telomere length. Without enzyme-cutting sites in the telomeric and subtelomeric regions, TRF is always considered as "gold standard" method (Tarik et al., 2018). For a large number of samples, PCR-based assays (including quantitative PCR, monochromatic multiplex quantitative PCR (MMqPCR) and absolute telomere length (aTL) quantification were applied to telomere length assessment. Unlike the assays described above, the substrate for quantitative-FISH (Q-FISH) is cells rather than DNA (Montpetit et al., 2014).

There are two mechanisms for telomere shortening, one of which is due to the inhibition of telomerase, resulting in incomplete DNA replication at the end of the chromosome, i.e., "end replication problem" (Guan et al., 2013). Cancer cells can also get rid of the limitations of aging by raising telomerase to maintain telomere levels (Shay and Bacchetti, 1997). In some cases, telomere length can be maintained by another mechanism,

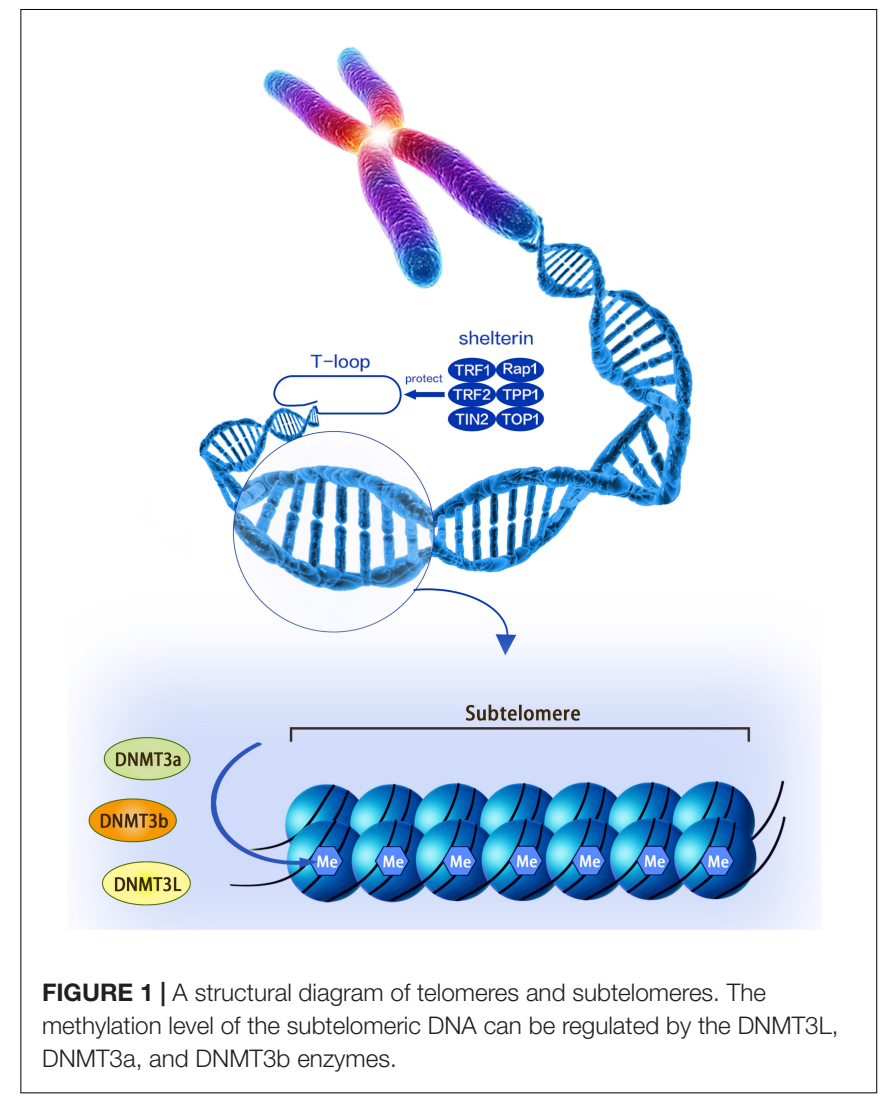

namely alternative lengthening of telomeres (ALT), which is based on homologous recombination-mediated DNA replication in the telomere sequence (Lee et al., 2009).

The somatic telomeres in the peripheral blood of the elderly are shorter than those in the young (Ye et al., 2018). In addition, telomere shortening is also accelerated by various pathophysiological conditions, including physical stress and disease state (Maeda et al., 2012). For example, according to the results of Kheirollahi, higher grade meningiomas and astrocytoma tumors show more heterogeneity in telomere length (Kheirollahi et al., 2011). Besides, telomere length is shortened and triggers chromosome instability in the early development of brain tumor (Nasser and Mehdipour, 2018).

\section{SUBTELOMERES AND SUBTELOMERIC DNA METHYLATION}

The vicinity of the telomeres is the so-called subtelomeric region, which is defined as $500 \mathrm{~kb}$ of each autosomal arm terminal (Macina et al., 1994). In 2004, Susann et al. discovered a subtelomeric DNA fragment called X-region in Hela cells. The researchers sequenced human subtelomeric sequences and found 941 transcripts, including 214 single-copy genes (Riethman et al., 2004). Both telomere and subtelomeric sequences have highdensity DNA repeats, but telomeres do not contain genes or CpG sequences, while subtelomeres contain small amounts of genes as well as high-density CpG sequences (Blasco, 2007). The 
telomeric DNA and their related proteins play an important role in genome stability and chromosome replication. Similarly, subtelomeric DNA fragments can partially regulate key biological activities, including cell cycle regulation, cell senescence and immortalization, movement and localization of chromosomes within the nucleus, and transcription of subtelomeric genes (Riethman et al., 2005). For example, subtelomeric abnormalities may have significant effects on idiopathic mental retardation and several mental retardation syndromes (Hila et al., 2009). Previous studies have shown that $6 \mathrm{p}$ subtelomeric deletions have occurred in growth stunting or mental retardation (DeScipio, 2007; Nakane et al., 2013). In addition, Wu et al. (2010) found that 16 patients with growth stunting or mental retardation had several deletions at 14 different subtelomeric regions (1p, 2p, $4 \mathrm{p}, 6 \mathrm{p}, 7 \mathrm{p}, 7 \mathrm{q}, 8 \mathrm{p}, 9 \mathrm{p}, 10 \mathrm{p}, 11 \mathrm{q}, 14 \mathrm{q}, 15 \mathrm{q}, 16 \mathrm{p}$, and $22 \mathrm{q})$ and repeats at seven subtelomeric regions $(3 q, 4 p, 6 q, 7 p, 8 p, 12 p$ and $22 \mathrm{q})$. What's more, subtelomeric rearrangements are also the major cause of idiopathic mental retardation, growth stunting or retardation, and malignant blood system diseases (Ravnan et al., 2006; Tos et al., 2013; El-Hajj Ghaoui et al., 2017).

Recent studies have found that epigenetic modification acted as a bridge between genetic and environmental factors and played an important role in the occurrence and development of diseases (Lovinsky-Desir and Miller, 2012; Hu et al., 2017). The regulatory mechanisms of epigenetics mainly include DNA methylation, histone modification, non-coding RNA action, and etc. DNA methylation, as one of the important mechanisms of epigenetics, has many important physiological significances in vivo. Normal methylation is essential for maintaining cell growth and metabolism, while abnormal DNA methylation causes disease (such as tumors) (Vera et al., 2008). The apparent modification of subtelomeres has long been of concern to scientists, and in 1990, de novo methylation was first found at a subtelomeric repeat with the minimal (4 kb) in somatic cells (de Lange et al., 1990). The subtelomeric region is rich in $\mathrm{CpG}$ islands, so DNA methylation often occurs in this region. A systematic search revealed a rare DNA methyltransferase $3 \mathrm{~L}$ (DNMT3L) variant that may affect the structure or function of the corresponding DNMT protein associated with subtelomeric hypomethylation (ElMaarri et al., 2009). During early development, the subtelomeric region was methylated by DNMT3b (Walton et al., 2011; SimoRiudalbas et al., 2015). At a critical stage in human embryonic development, subtelomeric DNA methylation may be necessary for maintaining normal telomere length (Yehezkel et al., 2013).

\section{SUBTELOMERIC DNA METHYLATION AND TELOMERE LENGTH}

Subtelomeric methylation is thought to be closely related to telomere length. Focusing on nucleotide sequences, the subtelomere has a high density of $\mathrm{CpG}$ sequences that are susceptible to methylation of DNMT (including DNMT1, DNMT3a, and DNMT3b) (Figure 1; Brock et al., 1999; Steinert et al., 2004; Blasco, 2007). Past reports indicated that the subtelomeric region in mice was largely methylated and this epigenetic modification was reduced in DNMT-deficient embryonic stem (ES) cells. Interestingly, this genetically deficient cell significantly prolonged telomeres compared to wild-type controls (Gonzalo et al., 2006). The following experiments demonstrated that this phenomenon was caused by increased homologous recombination in the telomere sequence, whereas the introduction of DNMTs $3 \mathrm{a}$ and $3 \mathrm{~b}$ into DNMTs-deficient cells restored telomere methylation and reduced homologous recombination of telomere sequences (Gonzalo et al., 2006). Therefore, DNA methylation in the subtelomeric repeat region has the effect of inhibiting telomere homologous recombination and may affect the ALT mechanism (Gonzalo et al., 2006). Similarly, as telomeres shorten, subtelomeric chromatin also undergoes a conformational transition to a more commonly stained state, accompanied by decreased methylation and increased acetylation in telomerase knockout mouse cells. This process is thought to promote the recruitment of telomerase (Benetti et al., 2007; Blasco, 2007). Another mouse model found that there was always more dense methylation in the telomerepositive cells compared to normal cells. At the same time, telomerase-positive cells can promote telomerase maintenance of telomere length by reducing the level of telomere-containing repeat RNA (TERRA), the transcription of which originates from the subtelomere (Ng et al., 2009).

In recent years, scientists have begun to explore the association between methylation of specific subtelomeric CpG sites, telomere length and gene expression. Buxton's study found that methylation at many gene promoter regions distributed in the subtelomeric region was positively correlated with telomere length in human leukocyte DNA. And shorter telomeres are significantly associated with decreased methylation levels at most of these sites. Therefore, the authors speculated that with the shortening of telomeres, the methylation levels of the subtelomeric regions of many gene promoters may change, which in turn may lead to changes in gene expression and increase the risk of age-related diseases (Buxton et al., 2014). In addition, Choudhury et al. (2016) used optogenetics to increase DNA methylation at specific subtelomeric $\mathrm{CpG}$ sites, resulting in a gradual increase in telomere length during replication of three generations of HeLa cells.

\section{SUBTELOMERIC DNA METHYLATION AND AGING-RELATED DISEASES}

In epidemiological studies, individuals with shorter telomeres have a higher mortality rate, almost twice as many as those with longer telomeres (Goglin et al., 2016). A large number of previous studies have shown that short telomere and telomere dysfunction were associated with many diseases. For example, a shorter telomere length may indicate a higher risk of agingrelated disease in patients (Herrmann et al., 2018).

It has been reported that abnormal epigenetic changes in subtelomeric CpG islands affected several biological pathways or induced cell reprogramming due to induction of methylation or hydroxymethylation, however, the specific mechanisms still need to be explored (Wang et al., 2013). Previous studies have shown that epigenetic modification of subtelomere had a certain 
TABLE 1 | The correlation between subtelomeric methylation and telomere length in aging-related diseases.

\begin{tabular}{|c|c|c|c|c|}
\hline Disease & $\begin{array}{l}\text { The location of } \\
\text { subtelomeric region }\end{array}$ & $\begin{array}{l}\text { The methylation status of } \\
\text { subtelomeric region }\end{array}$ & $\begin{array}{l}\text { The correlation between } \\
\text { subtelomeric methylation and } \\
\text { telomere length }\end{array}$ & Reference \\
\hline Glioblastomas & Chr. 4q (D4Z4) & Demethylation & NA & Cadieux et al., 2006 \\
\hline Glioblastomas & Chr. 10q & Hypomethylation & NA & Cadieux et al., 2006 \\
\hline Lung cancer & Chr. 8p & Hypomethylation & NA & Rauch et al., 2008 \\
\hline Hepatocellular carcinoma & Chr. $7 q$ & Hypomethylation & Irrelevant & Oh et al., 2011 \\
\hline Hepatocellular carcinoma & Chr. 18p & Hypermethylation & Negative & Oh et al., 2011 \\
\hline Hepatocellular carcinoma & Chr. $21 \mathrm{q}$ & Hypermethylation & Positive & On et al., 2011 \\
\hline Astrocytoma & Chr. $2 p$ & Hypermethylation & NA & Lyke et al., 2012 \\
\hline Nasopharyngeal carcinoma & Chr. 4q35 (D4Z4) & Demethylation & Positive & Zhang et al., 2014 \\
\hline Glioblastomas & Chr. $8 q$ & Hypermethylation & NA & Choudhury et al., 2015 \\
\hline Glioblastomas & Chr. $21 \mathrm{q}$ & Hypermethylation & NA & Choudhury et al., 2015 \\
\hline Glioblastomas & Chr. XpYp & Hypermethylation & NA & Choudhury et al., 2015 \\
\hline Gallbladder carcinoma & Chr. 4q35 (D4Z4) & Hypermethylation & Irrelevant & Poojary et al., 2016 \\
\hline Alzheimer's disease & NA & Hypermethylation & NA & Guan et al., 2013 \\
\hline Parkinson's disease & NA & Hypermethylation & Negative & Maeda et al., 2009 \\
\hline Hypertension & NA & Hypermethylation & Negative & Maeda et al., 2011b \\
\hline
\end{tabular}

NA: not available.

effect on the regulation of telomere length (Table 1). And the mouse-based model described above also suggested a conserved link between telomere length and the epigenetic state of the subtelomere. Therefore, we hypothesized that subtelomeric DNA methylation may cause aging disease by affecting telomere length.

As the elderly population increases, the incidence of agingrelated diseases is increasing, such as cancer, cardiovascular disease (CVD), type 2 diabetes (T2DM), neurodegenerative diseases, osteoporosis and premature aging. The consumption of telomeres is related to the aging process and to the condition of senile diseases. At the same time, the process of aging also affects the methylation status of the subtelomere.

\section{CANCER}

The risk of developing cancer increases along with aging, and thus cancer is defined as one of the common age-related diseases (Garrido and Djouder, 2017). One of the causes of cancer is the widespread distribution of DNA methylation in genomic chromatin. These changes often affect the structure of chromatin, which may cause abnormal expression of some oncogenes, leading to carcinogenesis (Klutstein et al., 2016; Li et al., 2018). Epigenetic modification of subtelomere can also lead to the similar consequences.

A large number of studies have validated the relationship between subtelomeric DNA methylation and the risk and prognosis of cancer. Poojary et al. (2016) found that in early gallbladder cancer, a chromosome $4 \mathrm{q} 35$ subtelomeric sequence (D4Z4) was hypermethylated and the telomere length was significantly shortened. However, no correlation was found between subtelomeric methylation and telomere length. In gliomas, their subtelomeric DNA methylation levels were consistently higher than in the control group, especially in the subtelomeric regions of chromosomes $8 \mathrm{q}, 21 \mathrm{q}$, and
XpYp, regardless of patient age and tumor grade (Choudhury et al., 2015). Zhang et al. (2014) evaluated the role of demethylation drug (5-AZA) in nasopharyngeal carcinoma cells (CNE, CNE1, CNE2, and 5-8F). They found that 5-AZA induced significant demethylation and inhibited the expression of the human telomerase catalytic subunit (hTERT), which reduced telomerase activity and significantly shortened telomere length. In nasopharyngeal carcinoma, demethylation of the chromosome 4q35 subtelomeric region (D4Z4) may result in decreased expression of hTERT and shortening of telomeres. However, in the demethylation experiment of malignant glioma cell lines, telomere length did not change with changes in subtelomeric DNA methylation (Choudhury et al., 2015). Yan et al. (2012) studied the genome-wide activity of decitabine in acute myeloid leukemia and found that decitabine preferred to demethylate the $\mathrm{CpG}$ islands in subtelomeres. And decitabine had different activities in different chromosomal regions, which provided a theoretical basis for demethylating drugs to treat diseases (Yan et al., 2012). In addition, Myung et al. found no significant association between telomere length and subtelomeric DNA methylation in human tumor cells by MSP and pyrosequencing (Lee et al., 2009).

High-throughput whole-genome sequencing has shown significantly hypomethylated subtelomeric regions on chromosomes $4 \mathrm{q}$ and $10 \mathrm{q}$ for malignant glioma (Cadieux et al., 2006) and chromosome 8p for lung cancer (Rauch et al., 2008). Liver cancer showed a higher methylation rate in the $18 \mathrm{p}$ and $21 \mathrm{q}$ subtelomeric regions compared to the control group. Interestingly, in patients with liver cancer, subtelomeric methylation at chromosome $18 \mathrm{p}$ was inversely correlated with telomere length; while subtelomeric methylation at chromosome 21q was positively correlated to telomere length (Oh et al., 2011). During the development of liver cancer, the telomere length is prolonged, accompanied by hypomethylated subtelomeric region at chromosome $7 \mathrm{q}$ and hypermethylated subtelomeric 
region at chromsome 21q (Oh et al., 2011). The methylation changes of different subtelomeres are different, which indicates that the relationship between human subtelomeric methylation and telomere length is complicated.

Transcription from subtelomeres produces TERRA, a natural inhibitor of telomerase activity (TA). In astrocytoma, DNA methylation of subtelomeric CpG island is associated with low expression of TERRA on the chromosome 2p (Sampl et al., 2012). This suggests that the expression of TERRA may be specific to the regulation of the subtelomeric methylation at one chromosome.

In prostate cancer, hypermethylation of the subtelomeric region $\mathrm{D} 4 \mathrm{Z} 4$ was found to be associated with the worse prognosis of the patients (Han et al., 2017). We hypothesize that subtelomeric DNA methylation may be a potential biomarker for tumor prognosis, and more experiments should be performed to validate this conjecture.

\section{NEURODEGENERATIVE DISEASES}

Alzheimer's disease (AD) and Parkinson's disease (PD) are the two most common chronic neurodegenerative diseases associated with aging. $\mathrm{AD}$ is characterized by a gradual decline in cognitive and memory functions. It is estimated that by 2050, the number of people affected by $\mathrm{AD}$ worldwide will reach 150 million $^{1}$. Guan et al. (2013) found that in patients with $\mathrm{AD}$, the subtelomere methylation of patients with short telomeres $(<4.4 \mathrm{~kb})$ was higher than that of patients with long telomeres. At the same time, they also found that leukocytes with short-telomeric DNA hypomethylation tend to be cleared more quickly from the peripheral blood of $\mathrm{AD}$ patients, resulting in normal telomere length in AD patients (Guan et al., 2012b). In addition, they also observed the role of antioxidant vitamin $\mathrm{E}$ in patients with $\mathrm{AD}$, and found that vitamin $\mathrm{E}$ could reduce the level of oxidative stress in $\mathrm{AD}$ patients, however, vitamin $\mathrm{E}$ had no significant effect on the telomere length of $\mathrm{AD}$ patients (Guan et al., 2012a).

Parkinson's disease (PD) is a neurodegenerative disease described as "shaking paralysis." Clinical features of PD include motor symptoms such as tremor, muscle stiffness, dyskinesia, postural instability, cognitive symptoms, and circadian rhythm disorders. It is estimated that among the 10 countries with the largest number of people in the world, the incidence of idiopathic Parkinson's disease in 2030 is expected to reach 8.7 to 9.3 million (Allyson Jones et al., 2012). Maeda et al. (2009) found a significant correlation between telomere shortening and subtelomeric methylation in PD. As PD-related neurodegeneration occurs, the state of telomeres and subtelomeres changes. Compared with the healthy control group, Japanese women with PD had a decrease in leukocytes with long telomeres and a proportional increase of hypomethylated subtelomeres in leukocytes with short telomeres (Maeda et al., 2012). PD also causes an increase in telomere fragility, which accelerates telomere shortening in leukocytes. Future experiments should be performed in more neurodegenerative diseases.

\footnotetext{
${ }^{1}$ https://www.alz.co.uk/research/world-report-2018
}

\section{CARDIOVASCULAR AND CEREBROVASCULAR DISEASES}

The incidence and mortality of CVD is extremely high, and the incidence will increase further in both developing and developed countries in the future. The pathophysiological features of CVD are endothelial dysfunction, vascular inflammation, atherosclerosis, fibrosis, and thrombosis. Studies have shown that telomere shortening was associated with the risk of coronary heart disease, myocardial infarction, heart failure, and stroke (Willeit et al., 2010). In hypertensive patients, the degree of subtelomeric methylation of long telomeres was often inversely correlated with telomere consumption (Maeda et al., 2011b). Researchers found that cardiac function was related to both telomere length and subtelomeric DNA methylation (Maeda et al., 2018). Maeda et al. (2013) also found that subtelomeres were hypomethylated and telomerase activity was decreased in senescent smooth muscle cells.

Cerebrovascular disease refers to various diseases of the blood vessels in brain, including cerebral atherosclerosis, thrombosis, stenosis, occlusion, cerebral arteritis, cerebral artery injury, cerebral aneurysm, intracranial vascular malformation, and cerebral arteriovenous fistula. Common features are caused by ischemia or hemorrhagic accidents in brain, resulting in disability or death of the patient. Maeda et al. (2010) studied the association of telomere length and subtelomeric DNA methylation with clinical laboratory data in patients with cerebral infarction and metabolic disorders. There was a significant correlation of fasting blood glucose, HbA1c, serum creatinine and urea nitrogen levels with telomere length and subtelomeric DNA hypermethylation. The physiological capacity of patients with cerebrovascular disease was positively correlated with telomere length, and the recovery of physiological ability was related to subtelomeric DNA hypermethylation (Maeda et al., 2011a).

Cardiovascular and cerebrovascular diseases are common diseases that seriously threaten the health of humans, especially middle-aged and elderly people. Previous studies have shown that cardiovascular and cerebrovascular diseases were significantly associated with subtelomeric DNA methylation and telomere length (Maeda et al., 2011b, 2018). In the future, subtelomeric DNA methylation should be investigated in other cardiovascular and cerebrovascular diseases (such as coronary heart disease, myocardial infarction, stroke, and etc.).

\section{CONCLUSION}

A growing body of experimental evidence indicates a significant change in the subtelomeric DNA methylation in age-related diseases. For example, subtelomeric DNA hypomethylation was found in lung cancer, malignant glioma, PD and other diseases, and subtelomeric DNA hypermethylation was found in diseases such as gallbladder cancer and AD. However, the methylation status of telomeres should be repeatedly demonstrated in large samples to arrive at credible conclusions.

The degree of subtelomeric methylation on different chromosomes or different arms in the same chromosome may 
not be the same. With the development of advanced technologies, FISH, an accurate molecular cytogenetic tool, uses fluorescently labeled DNA probes to identify microchromosomal changes that contain chromosomal rearrangements in the subtelomeric region (Meloni et al., 1993; Peterson et al., 2018). It reveals the diversity behavior of the p- and q-chromosomal arm signals. Recent FISHbased studies have shown that the reduced 5'RARA signal and the single PML-RARA fusion are located in the subterminal $\mathrm{q}$-arm and $\mathrm{p}$-arm regions of the rearranged chromosome 17, respectively (El-Hajj Ghaoui et al., 2017). Whether in a neoplastic disease or a non-cancerous disease, disease progression depends on the quantification and quality of the signal, and these signals differ between the $\mathrm{p}$ - and q-arms of each chromosome. Therefore, we should explore the quantity and quality of signals in different parts of each chromosome.

Furthermore, DNA methylation changes in the same subtelomere may not be similar in different age-related diseases. Interestingly, subtelomeric DNA hypomethylation at one site was not accompanied by a decrease in the global subtelomeric DNA methylation (Gonzalo et al., 2006). These results indicate DNA methylation changes in the subtelomeric loci may be specific to different disease. Unfortunately, up to now, there have been few studies on DNA methylation of specific subtelomeric regions, and this may be one of the next directions of subtelomeric researches. And subtelomeric DNA methylation in specific sites can be used as an indicator of age-related disease progression. Then, due to the complexity of age-related diseases, heterogeneity cannot be ignored, so multi-Omics should be used to find out the relevant mechanisms.

As telomere research continues to heat up, according to the present study, changes in telomere length associated with subtelomeric DNA methylation are considered to be important processes in the pathogenesis of aging-related diseases. In the previous concept, the association between subtelomeric DNA methylation and telomere length was not identical in similar diseases. In this review, the relationship between subtelomeric DNA methylation and telomere length is discussed. Some agerelated diseases were positively correlated with subtelomeric DNA methylation and telomere length, and negatively correlated with other age-related diseases, and the other parts were not significantly correlated. This makes it harder for us to study the correlation between telomere length and subtelomeric DNA methylation.

In addition, subtelomeric methylation and telomere length interact in some cases and have created a potential mechanism for several interactions. First, DNA methylation in the subtelomeric repeat region has the effect on inhibiting telomere homologous

\section{REFERENCES}

Allyson Jones, C., Wayne Martin, W. R., Wieler, M., King-Jesso, P., and Voaklander, D. C. (2012). Incidence and mortality of Parkinson's disease in older Canadians. Parkinsonism Relat. Disord. 18, 327-331. doi: 10.1016/j. parkreldis.2011.11.018

Benetti, R., Garcia-Cao, M., and Blasco, M. A. (2007). Telomere length regulates the epigenetic status of mammalian telomeres and subtelomeres. Nat. Genet. 39, 243-250. doi: 10.1038/ng1952 recombination and may affect ALT mechanisms to alter telomere length (Gonzalo et al., 2006). Second, telomerase-positive cells may alter the methylation level of the subtelomeric region by lowering TERRA levels, which may inhibit telomerase to maintain telomere length ( $\mathrm{Ng}$ et al., 2009). Third, as telomere shortens, subtelomeric chromatin is accompanied by decreased methylation, and telomerase knockout mice have increased acetylation, which is thought to be beneficial for telomerase recruitment (Benetti et al., 2007; Blasco, 2007). However, due to its complex etiology, the exact mechanism of age-related diseases is difficult to fully explain. Multi-Omics is used to increase the likelihood of a potential mechanism for determining the relationship between subtelomeric DNA methylation and telomere length through integration interactions and corresponding correlations. In addition, research on human transformation through in vivo and/or in vitro should be fully revealed in the future.

In addition, a large number of diseases are closely related to genomic risk factors, and attention to genomic levels provides a large number of clues for pathogenesis and drug targets. Researchers reduced subtelomeric DNA methylation by demethylating drugs, and hoped to negatively regulate the telomere length of tumor cells by DNA methylation, thereby reducing the stability of tumor cell genome and helping clinical diagnosis and treatment of tumors. Apart from demethylating drugs, regulation of telomere length by re-expression of telomerase or inhibition of telomerase may be a potential treatment for disease.

Finally, our review suggests that subtelomeric DNA methylation is highly associated with aging diseases. However, its role in the development of aging diseases is not totally unmasked, and therefore, the role of subtelomeric DNA methylation in the development of aging diseases will be further evaluated in the future.

\section{AUTHOR CONTRIBUTIONS}

$\mathrm{SD}$ and $\mathrm{HH}$ contributed to the conception, design, writing and final approval of the submitted version. $\mathrm{HH}$ and $\mathrm{BL}$ contributed to the revision of manuscript.

\section{FUNDING}

The research was supported by K. C. Wong Magna Fund in Ningbo University.

Blasco, M. A. (2007). The epigenetic regulation of mammalian telomeres. Nat. Rev. Genet. 8, 299-309. doi: 10.1038/nrg2047

Brock, G. J., Charlton, J., and Bird, A. (1999). Densely methylated sequences that are preferentially localized at telomere-proximal regions of human chromosomes. Gene 240, 269-277. doi: 10.1016/S0378-1119(99)00442-4

Buxton, J. L., Suderman, M., Pappas, J. J., Borghol, N., McArdle, W., Blakemore, A. I., et al. (2014). Human leukocyte telomere length is associated with DNA methylation levels in multiple subtelomeric and imprinted loci. Sci. Rep. 4:4954. doi: $10.1038 /$ srep04954 
Cadieux, B., Ching, T. T., VandenBerg, S. R., and Costello, J. F. (2006). Genomewide hypomethylation in human glioblastomas associated with specific copy number alteration, methylenetetrahydrofolate reductase allele status, and increased proliferation. Cancer Res. 66, 8469-8476. doi: 10.1158/0008-5472. CAN-06-1547

Choudhury, S. R., Cui, Y., Milton, J. R., Li, J., and Irudayaraj, J. (2015). Selective increase in subtelomeric DNA methylation: an epigenetic biomarker for malignant glioma. Clin. Epigenet. 7, 1-11. doi: 10.1186/s13148-015-0140-y

Choudhury, S. R., Cui, Y., Narayanan, A., Gilley, D. P., Huda, N., Lo, C. L., et al. (2016). Optogenetic regulation of site-specific subtelomeric DNA methylation. Oncotarget 7, 50380-50391. doi: 10.18632/oncotarget.10394

de Lange, T., Shiue, L., Myers, R. M., Cox, D. R., Naylor, S. L., Killery, A. M., et al. (1990). Structure and variability of human chromosome ends. Mol. Cell. Biol. 10, 518-527. doi: 10.1128/MCB.10.2.518

DeScipio, C. (2007). The 6p subtelomere deletion syndrome. Am. J. Med. Genet. C Semin. Med. Genet. 145C, 377-382. doi: 10.1002/ajmg.c.30156

El-Hajj Ghaoui, R., St Heaps, L., Hung, D., Nagabushan, S., Harris, C., Mirochnik, O., et al. (2017). A paediatric acute promyelocytic leukaemia patient harbouring a cryptic pml-rara insertion due to a complex structural chromosome 17 rearrangement. Cytogenet. Genome Res. 153, 181-189. doi: $10.1159 / 000487000$

El-Maarri, O., Kareta, M. S., Mikeska, T., Becker, T., Diaz-Lacava, A., Junen, J., et al. (2009). A systematic search for DNA methyltransferase polymorphisms reveals a rare DNMT3L variant associated with subtelomeric hypomethylation. Hum. Mol. Genet. 18, 1755-1768. doi: 10.1093/hmg/ ddp088

Garrido, A., and Djouder, N. (2017). NAD(+) deficits in age-related diseases and cancer. Trends Cancer 3, 593-610. doi: 10.1016/j.trecan.2017.06.001

Goglin, S. E., Farzaneh-Far, R., Epel, E. S., Lin, J., Blackburn, E. H., and Whooley, M. A. (2016). Change in leukocyte telomere length predicts mortality in patients with stable coronary heart disease from the heart and soul study. PLoS One 11:e0160748. doi: 10.1371/journal.pone.0160748

Gonzalo, S., Jaco, I., Fraga, M. F., Chen, T., Li, E., Esteller, M., et al. (2006). DNA methyltransferases control telomere length and telomere recombination in mammalian cells. Nat. Cell Biol. 8, 416-424. doi: 10.1038/ncb1386

Guan, J. Z., Guan, W. P., Maeda, T., and Makino, N. (2012a). Effect of vitamin $\mathrm{E}$ administration on the elevated oxygen stress and the telomeric and subtelomeric status in Alzheimer's disease. Gerontology 58, 62-69. doi: 10.1159/ 000327821

Guan, J. Z., Guan, W. P., Maeda, T., and Makino, N. (2012b). The subtelomere of short telomeres is hypermethylated in Alzheimer's Disease. Aging Dis. 3, $164-170$.

Guan, J. Z., Guan, W. P., Maeda, T., and Makino, N. (2013). Analysis of telomere length and subtelomeric methylation of circulating leukocytes in women with Alzheimer's disease. Aging Clin. Exp. Res. 25, 17-23. doi: 10.1007/s40520-0130006-0

Han, Y., Xu, J., Kim, J., Wu, X., and Gu, J. (2017). Methylation of subtelomeric repeat D4Z4 in peripheral blood leukocytes is associated with biochemical recurrence in localized prostate cancer patients. Carcinogenesis 38, 821-826. doi: 10.1093/carcin/bgx064

Harley, C. B., Futcher, A. B., and Greider, C. W. (1990). Telomeres shorten during ageing of human fibroblasts. Nature 345, 458-460. doi: 10.1038/345458a0

Herrmann, M., Pusceddu, I., Marz, W., and Herrmann, W. (2018). Telomere biology and age-related diseases. Clin. Chem. Lab. Med. 56, 1210-1222. doi: $10.1515 / \mathrm{cclm}-2017-0870$

Hila, L., Tebourbi, H., Abaied, L., Rejeb, I., Ben Jemaa, L., and Chaabouni, H. (2009). MLPA subtelomere analysis in Tunisian mentally retarded patients. Biochem. Genet. 47, 727-733. doi: 10.1007/s10528-009-9271-1

Hu, H., Chen, X., Wang, C., Jiang, Y., Li, J., Ying, X., et al. (2017). The role of TFPI2 hypermethylation in the detection of gastric and colorectal cancer. Oncotarget 8, 84054-84065. doi: 10.18632/oncotarget.21097

Kheirollahi, M., Mehrazin, M., Kamalian, N., and Mehdipour, P. (2011). Alterations of telomere length in human brain tumors. Med. Oncol. 28, 864870. doi: 10.1007/s12032-010-9506-3

Kim, N. W., Piatyszek, M. A., Prowse, K. R., Harley, C. B., West, M. D., Ho, P. L., et al. (1994). Specific association of human telomerase activity with immortal cells and cancer. Science 266, 2011-2015. doi: 10.1126/science.7605428
Kim, N. W., and Wu, F. (1997). Advances in quantification and characterization of telomerase activity by the telomeric repeat amplification protocol (TRAP). Nucleic Acids Res. 25, 2595-2597. doi: 10.1093/nar/25.13.2595

Klutstein, M., Nejman, D., Greenfield, R., and Cedar, H. (2016). DNA methylation in cancer and aging. Cancer Res. 76, 3446-3450. doi: 10.1158/0008-5472.CAN15-3278

Lee, M. E., Rha, S. Y., Jeung, H. C., Chung, H. C., and Oh, B. K. (2009). Subtelomeric DNA methylation and telomere length in human cancer cells. Cancer Lett. 281, 82-91. doi: 10.1016/j.canlet.2009.02.031

Li, B., Pan, R., Zhou, C., Dai, J., Mao, Y., Chen, M., et al. (2018). SMYD3 promoter hypomethylation is associated with the risk of colorectal cancer. Future Oncol. 14, 1825-1834. doi: 10.2217/fon-2017-0682

Lovinsky-Desir, S., and Miller, R. L. (2012). Epigenetics, asthma, and allergic diseases: a review of the latest advancements. Curr. Allergy Asthma Rep. 12, 211-220. doi: 10.1007/s11882-012-0257-4

Lyke, K. E., Dabo, A., Arama, C., Daou, M., Diarra, I., Wang, A., et al. (2012). Reduced T regulatory cell response during acute Plasmodium falciparum infection in Malian children co-infected with Schistosoma haematobium. PLoS One 7:e31647. doi: 10.1371/journal.pone.0031647

Macina, R. A., Negorev, D. G., Spais, C., Ruthig, L. A., Hu, X. L., and Riethman, H. C. (1994). Sequence organization of the human chromosome $2 q$ telomere. Hum. Mol. Genet. 3, 1847-1853. doi: 10.1093/hmg/3.10.1847

Maeda, T., Guan, J. Z., Koyanagi, M., Higuchi, Y., and Makino, N. (2012). Agingassociated alteration of telomere length and subtelomeric status in female patients with Parkinson's disease. J. Neurogenet. 26, 245-251. doi: 10.3109/ 01677063.2011.651665

Maeda, T., Guan, J. Z., Koyanagi, M., and Makino, N. (2013). Alterations in the telomere length distribution and the subtelomeric methylation status in human vascular endothelial cells under elevated temperature in culture condition. Aging Clin. Exp. Res. 25, 231-238. doi: 10.1007/s40520-013-0045-6

Maeda, T., Guan, J. Z., Oyama, J., Higuchi, Y., and Makino, N. (2009). Agingassociated alteration of subtelomeric methylation in Parkinson's disease. J. Gerontol. A Biol. Sci. Med. Sci. 64, 949-955. doi: 10.1093/gerona/glp070

Maeda, T., Horiuchi, T., and Makino, N. (2018). Epigenetic status of subtelomere of peripheral leukocytes corresponds to cardiographic parameters with a sex association. Geriatr. Gerontol. Int. 18, 1415-1419. doi: 10.1111/ggi.13472

Maeda, T., Oyama, J. I., Higuchi, Y., Arima, T., Mimori, K., and Makino, N. (2010). The correlation between the telomeric parameters and the clinical laboratory data in the patients with brain infarct and metabolic disorders. J. Nutr. Health Aging 14, 793-797. doi: 10.1007/s12603-010-0136-4

Maeda, T., Oyama, J., Higuchi, Y., Nishiyama, Y., Kudo, Y., Yamori, T., et al. (2011a). The physical ability of Japanese female elderly with cerebrovascular disease correlates with the telomere length and subtelomeric methylation status in their peripheral blood leukocytes. Gerontology 57, 137-143. doi: 10.1159/ 000314633

Maeda, T., Oyama, J. I., Sasaki, M., Arima, T., and Makino, N. (2011b). The correlation between the clinical laboratory data and the telomere length in peripheral blood leukocytes of Japanese female patients with hypertension. J. Nutr. Health Aging 15, 240-244. doi: 10.1007/s12603-010-0137-3

Meloni, A. M., Peier, A. M., Haddad, F. S., Powell, I. J., Block, A. W., Huben, R. P., et al. (1993). A new approach in the diagnosis and follow-up of bladder cancer. FISH analysis of urine, bladder washings, and tumors. Cancer Genet. Cytogenet. 71, 105-118. doi: 10.1016/0165-4608(93)90015-E

Montpetit, A. J., Alhareeri, A. A., Montpetit, M., Starkweather, A. R., Elmore, L. W., Filler, K., et al. (2014). Telomere length: a review of methods for measurement. Nurs. Res. 63, 289-299. doi: 10.1097/NNR.0000000000000037

Nakane, T., Kousuke, N., Sonoko, H., Yuko, K., Sato, H., Kubota, T., et al. (2013). $6 \mathrm{p}$ subtelomere deletion with congenital glaucoma, severe mental retardation, and growth impairment. Pediatr. Int. 55, 376-381. doi: 10.1111/j.1442-200X. 2012.03729.x

Nasser, M. M., and Mehdipour, P. (2018). Exploration of involved key genes and signaling diversity in brain tumors. Cell Mol. Neurobiol. 38, 393-419. doi: 10. 1007/s10571-017-0498-9

Ng, L. J., Cropley, J. E., Pickett, H. A., Reddel, R. R., and Suter, C. M. (2009). Telomerase activity is associated with an increase in DNA methylation at the proximal subtelomere and a reduction in telomeric transcription. Nucleic Acids Res. 37, 1152-1159. doi: 10.1093/nar/gkn1030 
Oh, B. K., Um, T. H., Choi, G. H., and Park, Y. N. (2011). Frequent changes in subtelomeric DNA methylation patterns and its relevance to telomere regulation during human hepatocarcinogenesis. Int. J. Cancer 128, 857-868. doi: $10.1002 /$ ijc. 25398

Peterson, J. F., Geddes, G. C., Basel, D. G., Schippman, D., Grignon, J. W. Jr., vanTuinen, $\mathrm{P}$, et al. (2018). Inheritance of a balanced $t(12 ; 20)(q 24.33 ; \mathrm{p} 12.2)$ and unbalanced $\operatorname{der}(13) \mathrm{t}(7 ; 13)(\mathrm{p} 21.3 ; \mathrm{q} 33.2)$ from a maternally derived double balanced translocation carrier. J. Pediatr. Genet. 7, 35-39. doi: 10.1055/s-00371605592

Poojary, S. S., Mishra, G., Gupta, S., Shrivastav, B. R., and Tiwari, P. K. (2016). Dysfunction of subtelomeric methylation and telomere length in gallstone disease and gallbladder cancer patients of North Central India. J. Hepatobiliary Pancreat. Sci. 23, 276-282. doi: 10.1002/jhbp.332

Rauch, T. A., Zhong, X., Wu, X., Wang, M., Kernstine, K. H., Wang, Z., et al. (2008). High-resolution mapping of DNA hypermethylation and hypomethylation in lung cancer. Proc. Natl. Acad. Sci. U.S.A. 105, 252-257. doi: 10.1073/pnas. 0710735105

Ravnan, J. B., Tepperberg, J. H., Papenhausen, P., Lamb, A. N., Hedrick, J., Eash, D., et al. (2006). Subtelomere FISH analysis of 11688 cases: an evaluation of the frequency and pattern of subtelomere rearrangements in individuals with developmental disabilities. J. Med. Genet. 43, 478-489. doi: 10.1136/jmg.2005. 036350

Riethman, H., Ambrosini, A., Castaneda, C., Finklestein, J., Hu, X. L., Mudunuri, U., et al. (2004). Mapping and initial analysis of human subtelomeric sequence assemblies. Genome Res. 14, 18-28. doi: 10.1101/gr.1245004

Riethman, H., Ambrosini, A., and Paul, S. (2005). Human subtelomere structure and variation. Chromosome Res. 13, 505-515. doi: 10.1007/s10577-005-0998-1

Sampl, S., Pramhas, S., Stern, C., Preusser, M., Marosi, C., and Holzmann, K. (2012). Expression of telomeres in astrocytoma WHO grade 2 to 4: terra level correlates with telomere length, telomerase activity, and advanced clinical grade. Transl. Oncol. 5, 56-65. doi: 10.1593/tlo.11202

Shay, J. W., and Bacchetti, S. (1997). A survey of telomerase activity in human cancer. Eur. J. Cancer 33, 787-791. doi: 10.1016/S0959-8049(97)00062-2

Simo-Riudalbas, L., Diaz-Lagares, A., Gatto, S., Gagliardi, M., Crujeiras, A. B., Matarazzo, M. R., et al. (2015). Genome-wide DNA methylation analysis identifies novel hypomethylated non-pericentromeric genes with potential clinical implications in icf syndrome. PLoS One 10:e0132517. doi: 10.1371/ journal.pone. 0132517

Steinert, S., Shay, J. W., and Wright, W. E. (2004). Modification of subtelomeric DNA. Mol. Cell. Biol. 24, 4571-4580. doi: 10.1128/MCB.24.10.4571-4580.2004

Tarik, M., Ramakrishnan, L., Sachdev, H. S., Tandon, N., Roy, A., Bhargava, S. K., et al. (2018). Validation of quantitative polymerase chain reaction with Southern blot method for telomere length analysis. Future Sci. OA 4:FSO282. doi: 10.4155/fsoa-2017-0115

Tos, T., Vurucu, S., Karkucak, M., Kozan, S., Gul, D., and Akin, R. (2013). Subtelomeric fish findings in Turkish patients with idiopathic mental retardation. Genet. Couns. 24, 259-264.
Vera, E., Canela, A., Fraga, M. F., Esteller, M., and Blasco, M. A. (2008). Epigenetic regulation of telomeres in human cancer. Oncogene 27, 6817-6833. doi: 10. 1038/onc.2008.289

Walton, E. L., Francastel, C., and Velasco, G. (2011). Maintenance of DNA methylation: dnmt3b joins the dance. Epigenetics 6, 1373-1377. doi: 10.4161/ epi.6.11.17978

Wang, T., Wu, H., Li, Y., Szulwach, K. E., Lin, L., Li, X., et al. (2013). Subtelomeric hotspots of aberrant 5-hydroxymethylcytosine-mediated epigenetic modifications during reprogramming to pluripotency. Nat. Cell Biol. 15, 700-711. doi: $10.1038 /$ ncb2748

Willeit, P., Willeit, J., Brandstatter, A., Ehrlenbach, S., Mayr, A., Gasperi, A., et al. (2010). Cellular aging reflected by leukocyte telomere length predicts advanced atherosclerosis and cardiovascular disease risk. Arterioscler. Thromb. Vasc. Biol. 30, 1649-1656. doi: 10.1161/ATVBAHA.110.205492

Wu, Y., Ji, T., Wang, J., Xiao, J., Wang, H., Li, J., et al. (2010). Submicroscopic subtelomeric aberrations in Chinese patients with unexplained developmental delay/mental retardation. BMC Med. Genet. 11:72. doi: 10.1186/1471-235 0-11-72

Yaku, H., Murashima, T., Miyoshi, D., and Sugimoto, N. (2013). A highly sensitive telomerase activity assay that eliminates false-negative results caused by PCR inhibitors. Molecules 18, 11751-11767. doi: 10.3390/molecules 181011751

Yan, P., Frankhouser, D., Murphy, M., Tam, H. H., Rodriguez, B., Curfman, J., et al. (2012). Genome-wide methylation profiling in decitabine-treated patients with acute myeloid leukemia. Blood 120, 2466-2474. doi: 10.1182/blood-2012-05429175

Ye, X., He, Z., Deng, P., Wei, Y., Zhou, J., and Huang, H. (2018). Characteristics of distribution and changes of telomere length in human. Zhong Nan Da Xue Xue Bao Yi Xue Ban 43, 945-949. doi: 10.11817/j.issn.1672-7347.2018.09.003

Yehezkel, S., Shaked, R., Sagie, S., Berkovitz, R., Shachar-Bener, H., Segev, Y., et al. (2013). Characterization and rescue of telomeric abnormalities in ICF syndrome type I fibroblasts. Front. Oncol. 3:35. doi: 10.3389/fonc.2013.00035

Zhang, Z. X., Wang, Y., Tao, Z. Z., Chen, S. M., Xiao, B. K., and Zhou, T. (2014). Subtelomeric demethylation deregulated hTERT expression, telomerase activity, and telomere length in four nasopharyngeal carcinoma cell lines. Cancer Biother. Radiopharm. 29, 289-294. doi: 10.1089/cbr.2013. 1581

Conflict of Interest Statement: The authors declare that the research was conducted in the absence of any commercial or financial relationships that could be construed as a potential conflict of interest.

Copyright (c) $2019 \mathrm{Hu}, \mathrm{Li}$ and Duan. This is an open-access article distributed under the terms of the Creative Commons Attribution License (CC BY). The use, distribution or reproduction in other forums is permitted, provided the original author(s) and the copyright owner(s) are credited and that the original publication in this journal is cited, in accordance with accepted academic practice. No use, distribution or reproduction is permitted which does not comply with these terms. 